\title{
A Woman with Salt-wasting Congenital Adrenal Hyperplasia Presenting with a Mucinous Ovarian Cystadenoma during Pregnancy
}

\author{
Yuji Kamata, Akinori Hayashi, Akifumi Ogawa, Raishi Ichikawa, \\ Tatsumi Moriya and Masayoshi Shichiri
}

\begin{abstract}
Women with congenital adrenal hyperplasia (CAH) caused by steroid 21-hydroxylase deficiency show reduced fertility, especially with the salt-wasting form. A 27 -year-old pregnant woman with this disease underwent laparotomy and oophorectomy to remove a multilocular ovarian tumor at 14 weeks of pregnancy. This proved to be a mucinous cystadenoma. Toward the third trimester, she presented with marked elevations of $17 \alpha$-hydroxyprogesterone and plasma renin activity. Careful management of endocrine and body fluid homeostasis allowed her to give birth to a healthy female infant with normal external genitalia. This case illustrates endocrinological parameters during pregnancy in a woman with classical salt-wasting CAH.
\end{abstract}

Key words: congenital adrenal hyperplasia, steroid 21-hydroxylase deficiency, plasma renin activity, $17 \alpha-$ hydroxyprogesterone, pregnancy

(Intern Med 50: 1981-1985, 2011)

(DOI: 10.2169/internalmedicine.50.5401)

\section{Introduction}

Congenital adrenal hyperplasia (CAH) caused by steroid 21-hydroxylase deficiency (21-OHD) is one of the most common congenital endocrine disorders. It is inherited as an autosomal recessive disorder with a reported prevalence of 1 : 19000 in Japan and slightly less than that in the USA and Europe $(1,2)$. Patients with the salt-wasting form of $\mathrm{CAH}$ manifest varying degrees of impaired cortisol and aldosterone synthesis and androgen excess caused by an abnormal CYP21A2 gene encoding the steroid 21-hydroxylase enzyme (1). Thus, women with severe, classical 21-OHD require lifelong replacement with glucocorticoids and often with mineralocorticoids as well to avoid adrenal insufficiency/androgen excess and sodium imbalance, respectively.

Accumulating lines of evidence indicate a low frequency of pregnancy among woman with CAH compared with their age-matched controls and this is especially true in the saltwasting form of classical CAH (3-7). The classical literature reveals very few deliveries by women with salt-wasting
$\mathrm{CAH}$ and detailed clinical information is not available for those cases (5, 8-10). More recent reports suggest improved fertility rates, largely thanks to earlier treatment of $\mathrm{CAH}$, improved compliance with therapy and surgical advances in genital reconstruction $(3,8,9)$. Thus, there will be greater opportunities in the future for physicians to encounter pregnant women with salt-wasting $\mathrm{CAH}$.

Normally pregnant women demonstrate a stimulated renin-angiotensin-aldosterone system and increased circulating testosterone levels as the gestation progresses $(11,12)$. This system plays a crucial role in the maintenance of fluid and electrolyte homeostasis and the plasma levels often provide an important clue to suspect a possible imbalance. However, the magnitude of impaired aldosterone synthesis and its influence on fluid and electrolytes homeostasis in adult women with 21-OHD remains unclarified. In pregnant women with salt-wasting $\mathrm{CAH}$, evaluations of salt-retaining balance and androgen status would provide information on the adequacy of corticosteroid supplementation, but even the essential question of whether corticosteroid doses should be increased during pregnancy remains unanswered $(5,13-15)$. 
(A)

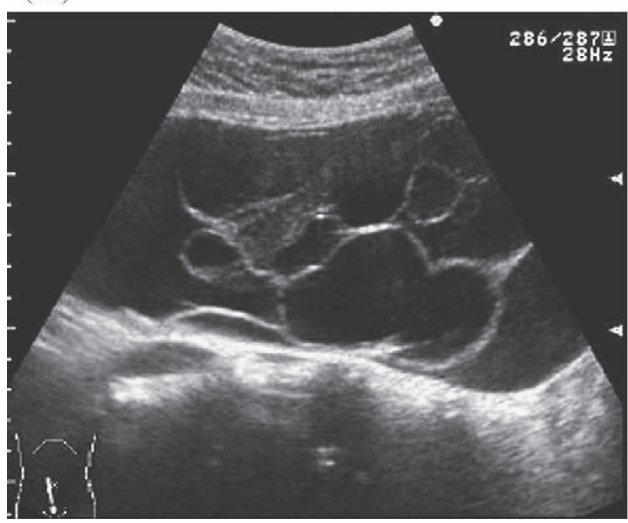

(B)

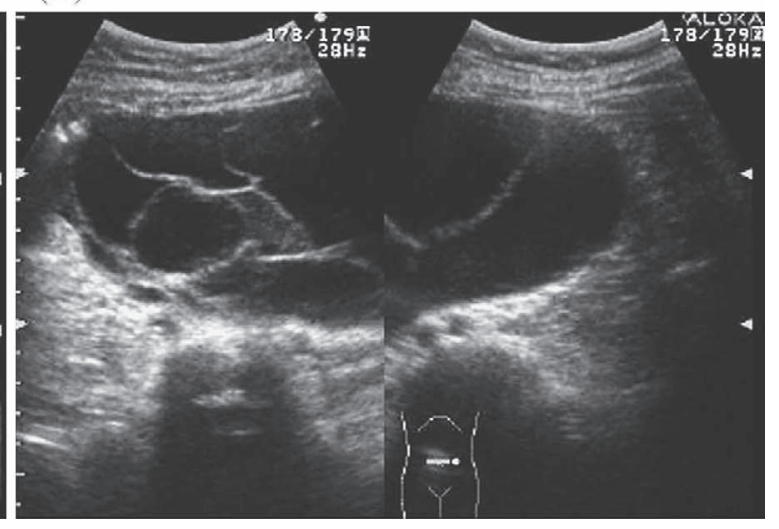

Figure 1. Abdominal ultrasonography findings at 12 weeks of gestation. (A) The left ovary showed a multilocular tumor with a septum seen by longitudinal scanning. (B) A multilocular left ovarian tumor measuring approximately $15.0 \times 7.6 \mathrm{~cm}$ was noted in a transverse scan.

(A)

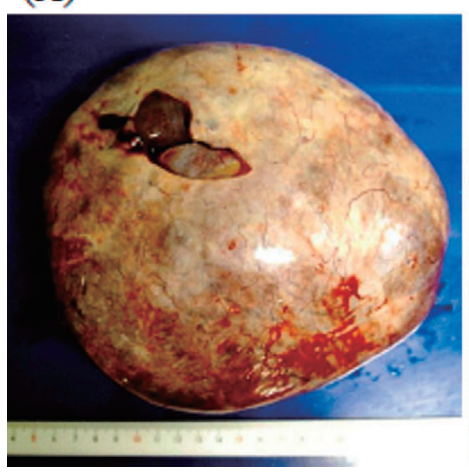

(B)

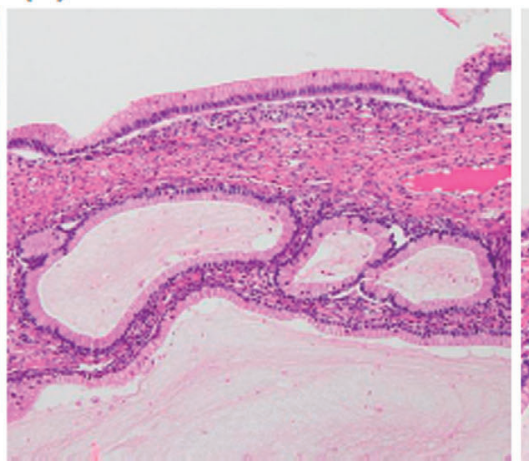

(C)

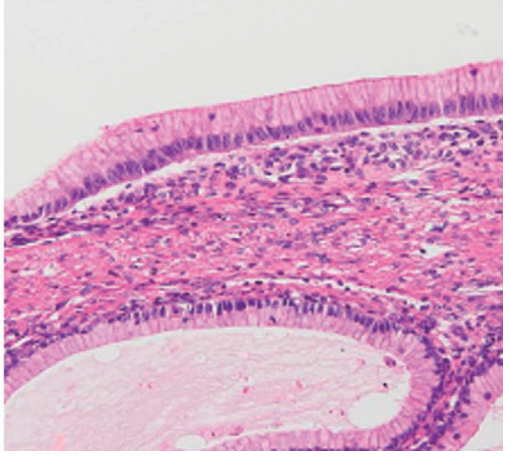

Figure 2. Surgical and pathological specimens of the left ovarian tumor. (A) Macroscopic observations of the ovarian tumor $(13.0 \times 9.5 \times 5.5 \mathrm{~cm}) .(\mathrm{B}, \mathrm{C})$ Histopathology using Hematoxylin and Eosin staining $(\times 40, \times 100)$. The neoplastic epithelium was composed of mucin-secreting columnar cells in a single row, with basal nuclei and interspersed goblet cells.

Such ambiguity, of course, derives from the scarcity of fundamental clinical information. We recently encountered a woman with classical salt-wasting $\mathrm{CAH}$ whose ovarian tumor was resected during pregnancy and for whom careful endocrinological and gynecological observation and management resulted in a successful delivery.

\section{Case Report}

A 27-year-old woman was referred to our hospital for adrenal steroid replacement therapy during pregnancy. She had been born weighing $3,220 \mathrm{~g}$ at a gestational age of 44 weeks and presented with salt-wasting, hyperkalemia and ambiguous genitalia, characterized by clitoromegaly, enlarged labia majora and pigmented external genitalia. She was diagnosed as having female pseudohermaphroditism caused by salt-wasting $\mathrm{CAH}$ with a $17 \alpha$-hydroxyprogesterone (17 $\alpha$-OHP) level of $35.85 \mathrm{ng} / \mathrm{mL}$ (normal range 0.38$4.8 \mathrm{ng} / \mathrm{mL}$ ). Her karyotype was 46 , XX. Treatment was initiated with hydrocortisone and fludrocortisone. Clitoridec- tomy and labial introitoplasty were performed at the age of three years. She experienced menarche at the age of 13 years. She has long been treated with $10 \mathrm{mg} /$ day hydrocortisone, $0.06 \mathrm{mg} /$ day fludrocortisone and $0.25 \mathrm{mg} / \mathrm{day}$ dexamethasone. In August 2009, she was referred to our hospital at seven weeks of pregnancy. Abdominal ultrasonography revealed a left multilocular ovarian tumor (Fig. 1A, B), for which laparotomy and oophorectomy were successfully performed at 14 weeks of pregnancy. Histopathology of the resected tumor $(13.0 \times 9.5 \times 5.5 \mathrm{~cm}$; Fig. $2 \mathrm{~A})$ revealed a mucinous cystadenoma (Fig. 2B, C). During the entire follow-up period, fluid and electrolyte balance, as well as, endocrine measures were carefully assessed and blood testing after the second trimester of pregnancy was performed with the patient placed in the right decubitus position. The $17 \alpha-\mathrm{OHP}$ levels elevated markedly toward the end of pregnancy, then returned to pregestational levels by 14 days following delivery, while androgen levels remained within the normal range (Fig. 3). ACTH levels were either suppressed or showed low normal values throughout pregnancy (Fig. 4). There was 


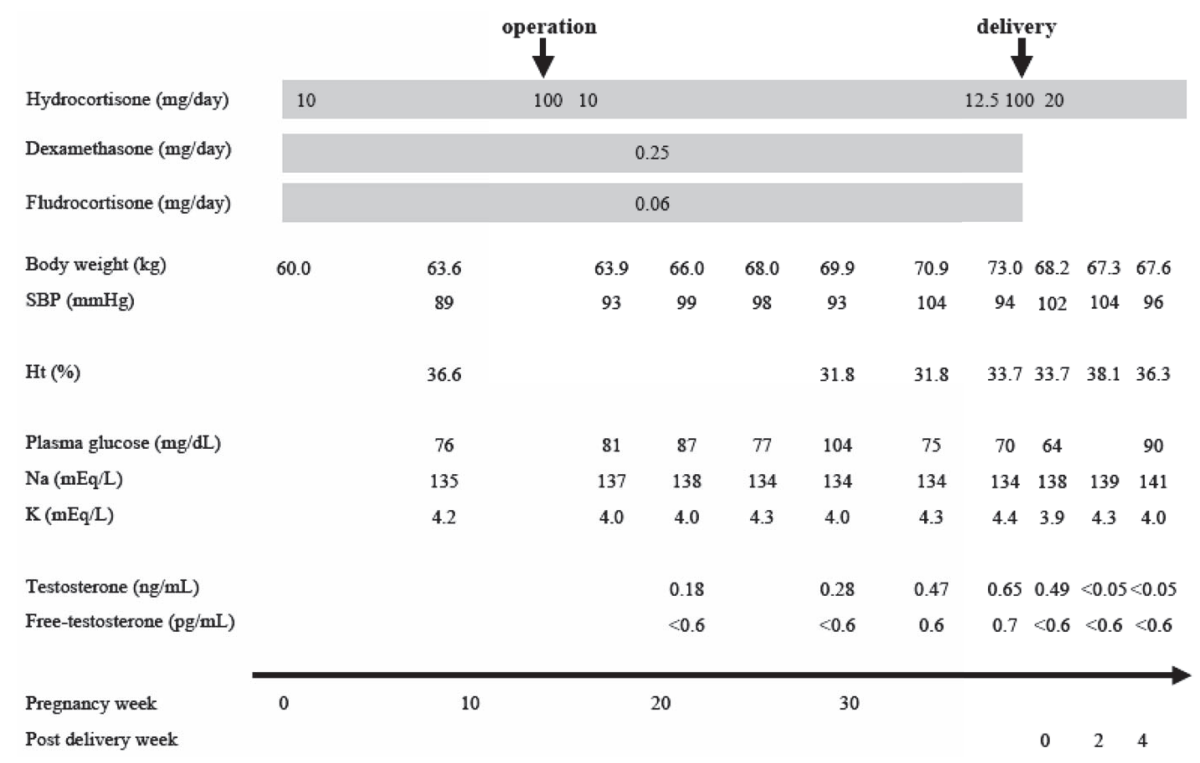

Figure 3. Biochemical, hematocrit and endocrine measures, blood pressure and body weight changes throughout pregnancy and after delivery.
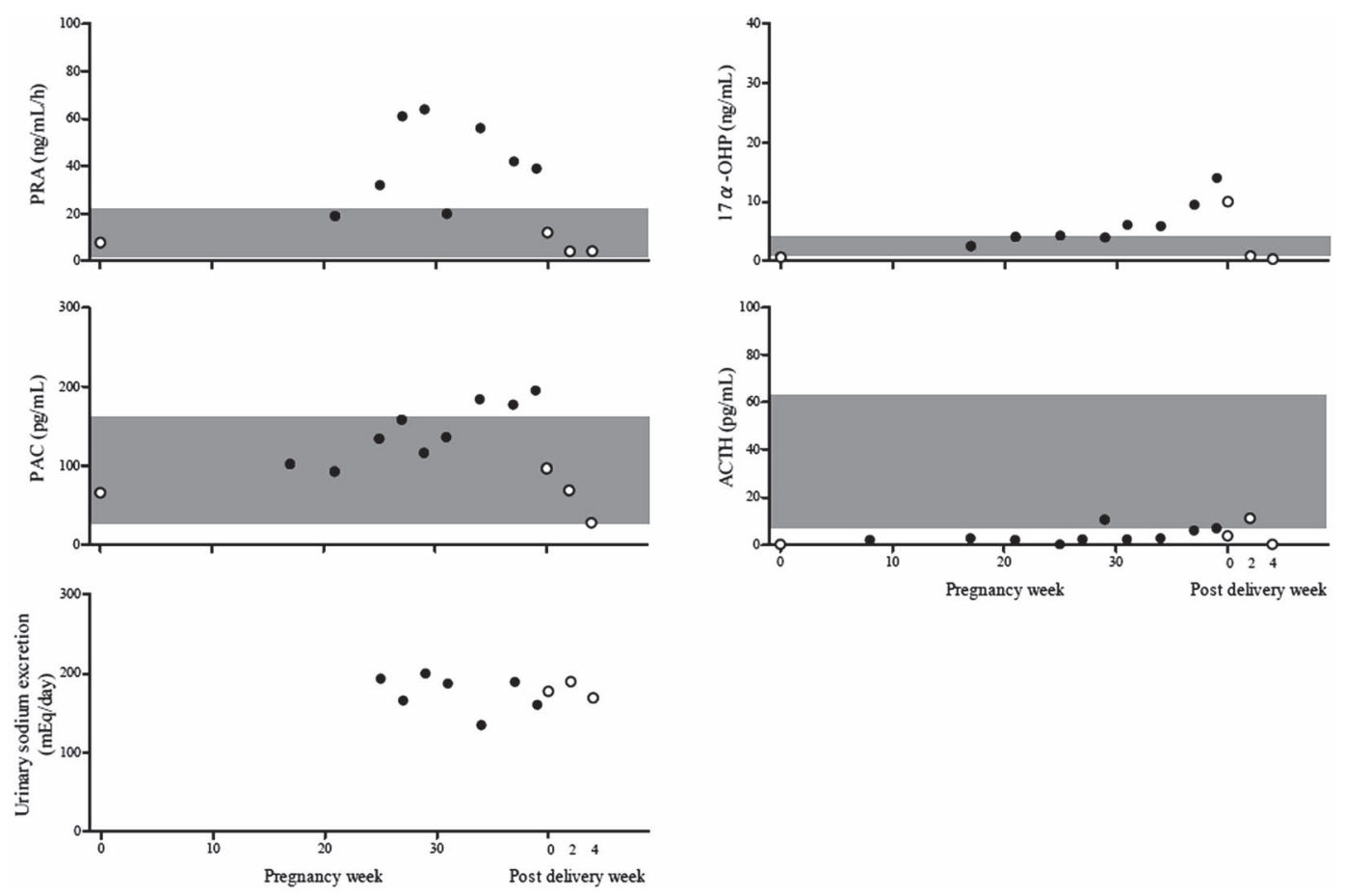

Figure 4. Changes in plasma renin activity (PRA), plasma aldosterone concentration (PAC), urinary sodium excretion, $17 \alpha$-hydroxyprogesterone (17 $\alpha-\mathrm{OHP})$ and ACTH during pregnancy and after delivery. Open circles: pre-pregnancy and post delivery values. Closed circles: data obtained during pregnancy. The painted areas show normal ranges for non-pregnant women. Urinary sodium excretion was estimated from the measured sodium and creatinine levels.

marked progressive elevation of plasma renin activity (PRA) through the third trimester until delivery (Fig. 4). She was carefully monitored to detect any clinical evidence of circulating volume depletion or overhydration, which turned out to be a needless anxiety. No signs of adrenal insufficiency such as hypotonia or hypoglycemia were seen throughout the pregnancy. Urinary sodium excretion, as estimated by the measured sodium and creatinine in casual urine specimens (16), was maintained constantly from the third trimester to delivery (Fig. 4), while urinary potassium excretion was $30-70 \mathrm{mEq} /$ day throughout. Fractional excretion of urate, which reflects extracellular fluid volume depletion (17), remained within the normal range (6.7-9.7\%) from the third trimester to delivery. At 29 weeks of pregnancy, 
human atrial natriuretic peptide and vasopressin levels showed normal values with $31.8 \mathrm{pg} / \mathrm{mL}$ (normal $<43.0 \mathrm{pg}$ / $\mathrm{mL}$ ) and $1.1 \mathrm{pg} / \mathrm{mL}$ (normal range, 0.3-3.5 pg/mL), respectively. At 40 weeks of pregnancy, she delivered a healthy $3.2 \mathrm{~kg}$ female neonate with normal external genitalia. The maternal hormone levels fell at four weeks postpartum (Fig. 3).

\section{Discussion}

Pregnant women with CAH can usually be maintained with the same glucocorticoid dosage as before pregnancy, but some patients with salt-wasting $\mathrm{CAH}$ require higher steroid doses to treat the symptoms of adrenal insufficiency (15). PRA or serum $17 \alpha$-OHP levels are regarded as indicators for adrenal steroid replacement and serve to help optimizing the steroid doses prescribed for such women. In normal pregnancy, serum $17 \alpha-\mathrm{OHP}$ increases during the second half of pregnancy and reaches approximately three-fold higher values at term, reflecting the production from fetal and maternal adrenal glands $(18,19)$. PRA and plasma aldosterone concentration (PAC) also show progressive increases contributed by a number of complicated factors including progesterone, arginine vasopressin, atrial natriuretic factor and prostaglandins (20). Among these, progesterone exerts natriuretic and hypotensive effects via its competition with mineralocorticoids (21). PRA and PAC were reported to show several-fold higher levels over non-pregnancy values, reaching as high as $15 \mathrm{ng} / \mathrm{mL} / \mathrm{h}$ and $800 \mathrm{pg} / \mathrm{mL}$ in third trimesters, respectively (22-24). Despite relatively stable PRA levels, the magnitude of increased aldosterone levels during the third trimester of pregnancy is more remarkable (25). In the present case, PAC showed only a marginal increase while the PRA was markedly elevated. It is reasonable to ascribe the markedly elevated PRA and contrasting very slight changes of PAC to the prevention of aldosterone production by steroid 21-hydroxylase deficiency. Our patient did not show any clear-cut evidence to suggest adrenal insufficiency throughout the pregnancy, despite the markedly elevated $17 \alpha$-OHP level and PRA during the third trimester.

Endocrinological monitoring to assess adrenal androgen suppression in the mother is recommended in pregnant woman with CAH. This is especially important when the fetus is female, because excessive intrauterine androgen exposure increases the risk of masculinization (15). Longitudinal studies indicate that circulating testosterone and sex hormone-binding protein levels are elevated during normal pregnancy $(12,19)$. Free testosterone levels might decrease early in pregnancy and increase later $(12,26)$. In the present case, the free testosterone levels were less than the assay sensitivity throughout pregnancy and this was likely to be the result of suppressed ACTH levels by dexamethasone therapy. Maternal ACTH levels may show progressive increases to as high as approximately two-fold as the placenta secretes larger amount of corticotropin-releasing hormone (CRH) into the maternal circulation (27). A previous report showed that ACTH levels in pregnant women were suppressed by betamethasone despite the elevated CRH (28). On the other hand, dexamethasone is transferred across the placenta without oxidation of the 11-hydroxyl group and provides long and effective suppression of adrenal androgen production, suppressing the fetal adrenal gland activity $(29,30)$. However, a previous case report suggested delivery of a normal neonate with dexamethasone treatment $(5,15)$. Therefore, it remains to be established whether pregnant women with salt-wasting $\mathrm{CAH}$ should be treated with dexamethazone.

Ovarian tumors in pregnancy are not unusual, with an estimated incidence of 1-2\% (31). For such women, particularly those showing tumor enlargement, ovariectomy is required to prevent an acute abdominal crisis caused by torsion, rupture, or hemorrhage of the tumor. Therefore, in the present case, we performed ovariectomy after confirmation of tumor growth in the second trimester of pregnancy. Histopathology showed it to be a mucinous cystadenoma, which accounts for approximately $10 \%$ among all ovarian tumors complicating pregnancy (32). Mucinous ovarian tumors are markedly heterogeneous, but they do not autonomously secrete hormones. They are characterized by the presence of mucins in some or all of the tumor cells, which resemble endocervical, gastric, or goblet cells. The exact incidence of ovarian tumors including the mucinous cystadenoma in women with $\mathrm{CAH}$ is yet undefined, although a number of reports indicate the development of polycystic ovaries in CAH women $(33,34)$. The frequency of adrenal rest tumor in women with CAH is less than in men (33). Polycystic ovaries and adrenal rest tumor are generally thought to result from intraovarian androgen excess and may be ACTH-dependent in women with $\mathrm{CAH}$. Therefore, it is reasonable to control ACTH levels using dexamethazone during marriageable age and to treat menstrual disorders, if any, in order to avoid the possible development of polycystic ovaries or adrenal rest tumor. However, since polycystic ovaries and adrenal rest tumors do develop independently of $\mathrm{ACTH}$ in women with $\mathrm{CAH}$, it is expected that clarification of the endocrinologic conditions in which women with $\mathrm{CAH}$ could avert the development of ovarian tumor including the mucinous cystadenoma.

In summary, we report a case of a pregnant woman with salt-wasting $\mathrm{CAH}$ who incidentally developed a mucinous cystadenoma of the ovary and successfully delivered a healthy female infant with normal external genitalia. Endocrinological monitoring throughout pregnancy in women with salt-wasting $\mathrm{CAH}$ is rarely reported, thus this case is relevant given the increasing likelihood that clinicians will encounter this situation.

The authors state that they have no Conflict of Interest (COI).

\section{References}

1. White PC, Speiser PW. Congenital adrenal hyperplasia due to 21- 
hydroxylase deficiency. Endocr Rev 21: 245-291, 2000.

2. van der Kamp HJ, Wit JM. Neonatal screening for congenital adrenal hyperplasia. Eur J Endocrinol 151: U71-U75, 2004.

3. Gastaud F, Bouvattier C, Duranteau L, et al. Impaired sexual and reproductive outcomes in women with classical forms of congenital adrenal hyperplasia. J Clin Endocrinol Metab 92: 1391-1396, 2007.

4. Jääskeläinen J, Hippeläinen M, Kiekara $O$, Voutilainen R. Child rate, pregnancy outcome and ovarian function in females with 21hydroxylase deficiency. Acta Obstet Gynecol Sand 79: 687-692, 2000.

5. Krone N, Wachter I, Stefanidou M, Roscher AA, Schwarz HP. Mothers with congenital adrenal hyperplasia and their children: outcome of pregnancy, birth and childhood. Clin Endocrinol (Oxf) 55: 523-529, 2001.

6. Mulaikal RM, Migeon CJ, Rock JA. Fertility rates in female patients with congenital adrenal hyperplasia due to 21-hydroxylase deficiency. N Engl J Med 316: 178-182, 1987.

7. Stikkelbroeck NM, Hermus AR, Braat DD, Otten BJ. Fertility in women with congenital adrenal hyperplasia due to 21-hydroxylase deficiency. Obstet Gynecol Surv 58: 275-284, 2003.

8. Hagenfeldt K, Janson PO, Holmdahl G, et al. Fertility and pregnancy outcome in women with congenital adrenal hyperplasia due to 21-hydroxylase deficiency. Hum Reprod 23: 1607-1613, 2008.

9. Lo JC, Grumbach MM. Pregnancy outcomes in women with congenital virilizing adrenal hyperplasia. Endocrinol Metab Clin North Am 30: 207-229, 2001.

10. Hoepffner W, Schulze E, Bennek J, Keller E, Willgerodt H. Pregnancies in patients with congenital adrenal hyperplasia with complete or almost complete impairment of 21-hydroxylase activity. Fertil Steril 81: 1314-1321, 2004.

11. Wilson M, Morganti AA, Zervoudakis I, et al. Blood pressure, the renin-aldosterone system and sex steroids throughout normal pregnancy. Am J Med 68: 97-104, 1980.

12. Kerlan V, Nahoul K, Le Martelot MT, Bercovici JP. Longitudinal study of maternal plasma bioavailable testosterone and androstanediol glucuronide levels during pregnancy. Clin Endocrinol (Oxf) 40: 263-267, 1994.

13. Amadori P. Reduced need of glucocorticoid therapy in a woman with congenital adrenal hyperplasia due to 21-hydroxylase deficiency during pregnancy. J Endocrinol Invest 29: 848-850, 2006.

14. Garner PR. Congenital adrenal hyperplasia in pregnancy. Semin Perinatal 22: 446-456, 1998.

15. Lo JC, Schwitzgebel VM, Tyrrell JB, et al. Normal female infants born of mothers with classic congenital adrenal hyperplasia due to 21-hydroxylase deficiency. J Clin Endocrinol Metab 84: 930-936, 1999.

16. Tanaka $T$, Okamura $T$, Miura $K$, et al. A simple method to estimate populational $24-\mathrm{h}$ urinary sodium and potassium excretion using a casual urine specimen. J Hum Hypertens 16: 97-103, 2002 .

17. Fushimi K, Shichiri M, Marumo F. Decreased fractional excretion of urate as an indicator of prerenal azotemia. Am J Nephrol $\mathbf{1 0}$
489-494, 1990.

18. Tulchinsky D, Simmer HH. Sources of plasma 17alpha-hydroxyprogesterone in human pregnancy. J Clin Endocrinol Metab 35: 799-808, 1972.

19. O'Leary P, Boyne P, Flett P, Beilby J, James I. Longitudinal assessment of changes in reproductive hormones during normal pregnancy. Clin Chem 37: 667-672, 1991.

20. de Jong CL, Dekker GA, Sibai BM. The renin-aldosterone system in preeclampsia. A review. Clin Perinatol 18: 683-711, 1991.

21. Oelkers WK. Effects of estrogens and progestogens on the reninaldosterone system and blood pressure. Steroids 61: 166-171, 1996.

22. August $P$, Lenz T, Ales KL. Longitudinal study of the reninangiotensin-aldosterone system in hypertensive pregnant women: deviations related to the development of superimposed preeclampsia. Am J Obstet Gynecol 163: 1612-1621, 1990.

23. Elsheikh A, Creatsas G, Mastorakos G, Milingos S, Loutradis D, Michalas S. The renin-aldosterone system during normal and hypertensive pregnancy. Arch Gynecol Obstet 264: 182-185, 2001.

24. Dörr HG, Heller A, Versmold HT. Longitudinal study of progestins, mineralocorticoids, and glucocorticoids throughout human pregnancy. J Clin Endocrinol Metab 68: 863-868, 1989.

25. Wu SJ, Tsai YL, Hsieh BS. Sequential changes in plasma renin activity and aldosterone level during pregnancy. J Formos Med Assoc 90: 932-935, 1991.

26. Bammann BL, Coulam CB, Jiang NS. Total and free testosterone during pregnancy. Am J Obstet Gynecol 137: 293-298, 1980.

27. Goland RS, Jozak S, Conwell I. Placental corticotropin-releasing hormone and the hypercortisolism of pregnancy. Am J Obstet Gynecol 171: 1287-1291, 1994.

28. Tropper PJ, Goland RS, Wardlaw SL, Fox HE, Frantz AG. Effects of betamethasone on maternal plasma corticotropin releasing factor, ACTH and cortisol during pregnancy. J Perinat Med 15: 221225, 1987.

29. Richards GE, Grumbach MM, Kaplan SL, Conte FA. The effect of long acting glucocorticoids on menstrual abnormalities in patients with virilizing congenital adrenal hyperplasia. J Clin Endocrinol Metab 47: 1208-1215, 1978.

30. Seckl JR, Chapman KE. Medical and physiological aspects of the 11 beta-hydroxysteroid dehydrogenase system. Eur J Biochem 249: 361-364, 1997.

31. Leiserowitz GS. Managing ovarian masses during pregnancy. Obstet Gynecol Surg 61: 463-470, 2006.

32. Duggan B, Muderspach LI, Roman LD, Curtin JP, d'Ablaing G 3rd, Morrow CP. Cervical cancer in pregnancy: reporting on planned delay in therapy. Obstet Gynecol 82: 598-602, 1993.

33. Stikkelbroeck NM, Hermus AR, Schouten D, et al. Prevalence of ovarian adrenal rest tumours and polycystic ovaries in females with congenital adrenal hyperplasia: results of ultrasonography and MR imaging. Eur Radiol 14: 1802-1806, 2004.

34. Hague WM, Adams J, Rodda C, et al. The prevalence of polycystic ovaries in patients with congenital adrenal hyperplasia and their close relatives. Clin Endcrinol (Oxf) 33: 501-510, 1990.

(C) 2011 The Japanese Society of Internal Medicine http://www.naika.or.jp/imindex.html 